\title{
Nanoparticle Movement: Plasmonic Forces and Physical Constraints
}

P.E. Batson ${ }^{1,2}$, A. Reyes-Coronado ${ }^{3}$, R.G. Barrera ${ }^{6}$, A. Rivacoba ${ }^{3,4,5}$, P.M. Echenique ${ }^{3,4,5}$, J. Aizpurua ${ }^{3,4}$

${ }^{1}$ Institute for Advanced Materials, Devices, and Nanotechnology, Rutgers University, NJ, ${ }^{2}$ IBM Thomas J. Watson Research Center, Yorktown Heights, NY, ${ }^{3}$ Donostia International Physics Center, San Sebastian, Spain, ${ }^{4}$ Centro de Física de Materiales CSIC-UPV/EHU, San Sebastian, Spain, ${ }^{5}$ Universidad del Pais Vasco (UPV)/EHU, Spain. ${ }^{6}$ Instituto de Física, Universidad Nacional Autónoma de México, Mexico City, Mexico

In previous work, we have explored the behavior of pairs of Au nanoparticles in the aberration corrected STEM, experimentally [1] and theoretically [2], showing that nanoparticle movement in the microscope can arise from forces driven by plasmonic fields excited by the passing electron beam. These forces belong to the same family of bonding forces that hold all materials together, including London Dispersion forces, VanderWaals, Debye and other electrostatics based forces. They are central to the dynamic behavior that moves and orients molecules in self-assembly, that transport complex molecules in biology, and that enable DNA and protein measurement using nanopore structures.

Looking in more detail, many different kinds of behavior emerge. These include 1) coalescence, discussed previously [1], 2) separation, wherein nanoparticles are driven apart, 3) pushing or pulling of single nanoparticles, 4) atomic level directing of atoms, and 5) complex multiple particle behaviors. Theoretically, many different geometric combinations need to be tested. In particular, these will include composite structures: spheres embedded in dielectrics, in contact with conducting layers, near nanopores or protrusions, or immersed in liquids, particularly those having ionic character.

In Fig. 1, we show results of a calculation for the momentum transfer from a passing swift electron to a single sphere, compared with a pair of identical spheres [2]. For the single sphere, the calculation predicts a weakly attractive force for moderate distances - a few times the diameter of the sphere. At very short distances, the force reverses sign, resulting in a repulsive push rather than an attractive pull that is the intuitive result for an image charge mechanism. For a pair of spheres, with about $0.2-0.3 \mathrm{~nm}$ separation, neither sphere is pulled towards the electron beam. Instead, the two spheres are driven together, resulting in the coalescence.

In Fig. 2, we show a coalescent behavior that appears to be modified by atomic level constraints. In other observations, coalescence occurs almost explosively, within one frame, as the smaller particle detaches from the substrate under the induced fields. In this sequence, it is apparently separated from the larger particle by a barrier which is not apparent in the HAADF imaging. The barrier, however, apparently contains a small pore (arrow) that is found and exploited by the smaller particle as a way to transport material an atom at a time from the smaller particle to the larger particle. In this presentation, this and other observed behavior will be discussed with theoretical model calculations in the context of discovery of material transport mechanisms at the nanoscale. 
PEB acknowledges support for this work by the Basic Energy Sciences Division of the Department of Energy, Award \#DE-SC0005132.

[1] P. E. Batson, Micros. Microanal., 14 (2008) 89 - 97.

[2] A. Reyes-Coronado, R. G. Barrera, P. E. Batson, P. M. Echenique, A. Rivacoba, and J. Aizpurua, Phys. Rev. B, 82 (2010) 235429.

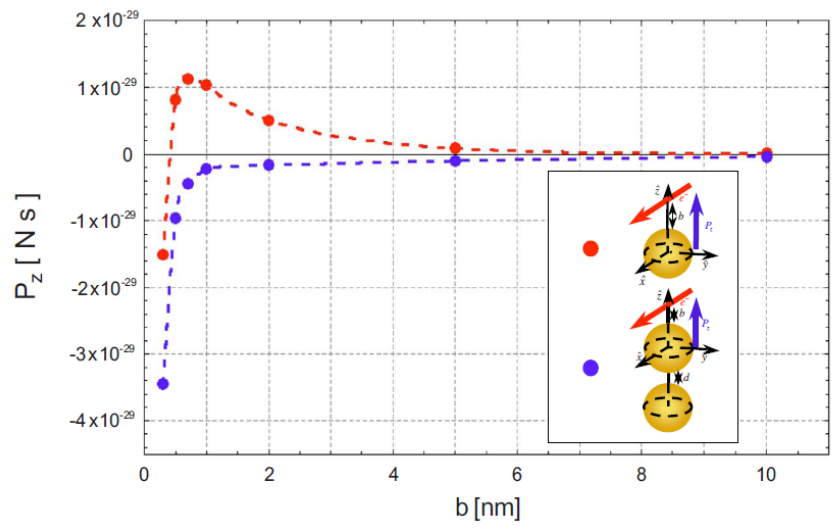

Fig. 1. Comparison of total momentum transfer along the $\mathrm{z}$ direction as a function of the impact parameter $b$, between a single small $(\mathrm{R}=1 \mathrm{~nm})$ gold particle (red curve) and the top particle in a dimer of small $(\mathrm{R}=1$ $\mathrm{nm}$ ) gold identical particles separated by a distance $\mathrm{d}=0.25 \mathrm{~nm}$ (blue curve). Dashed lines are a guide to the eye. Reproduced from [2].

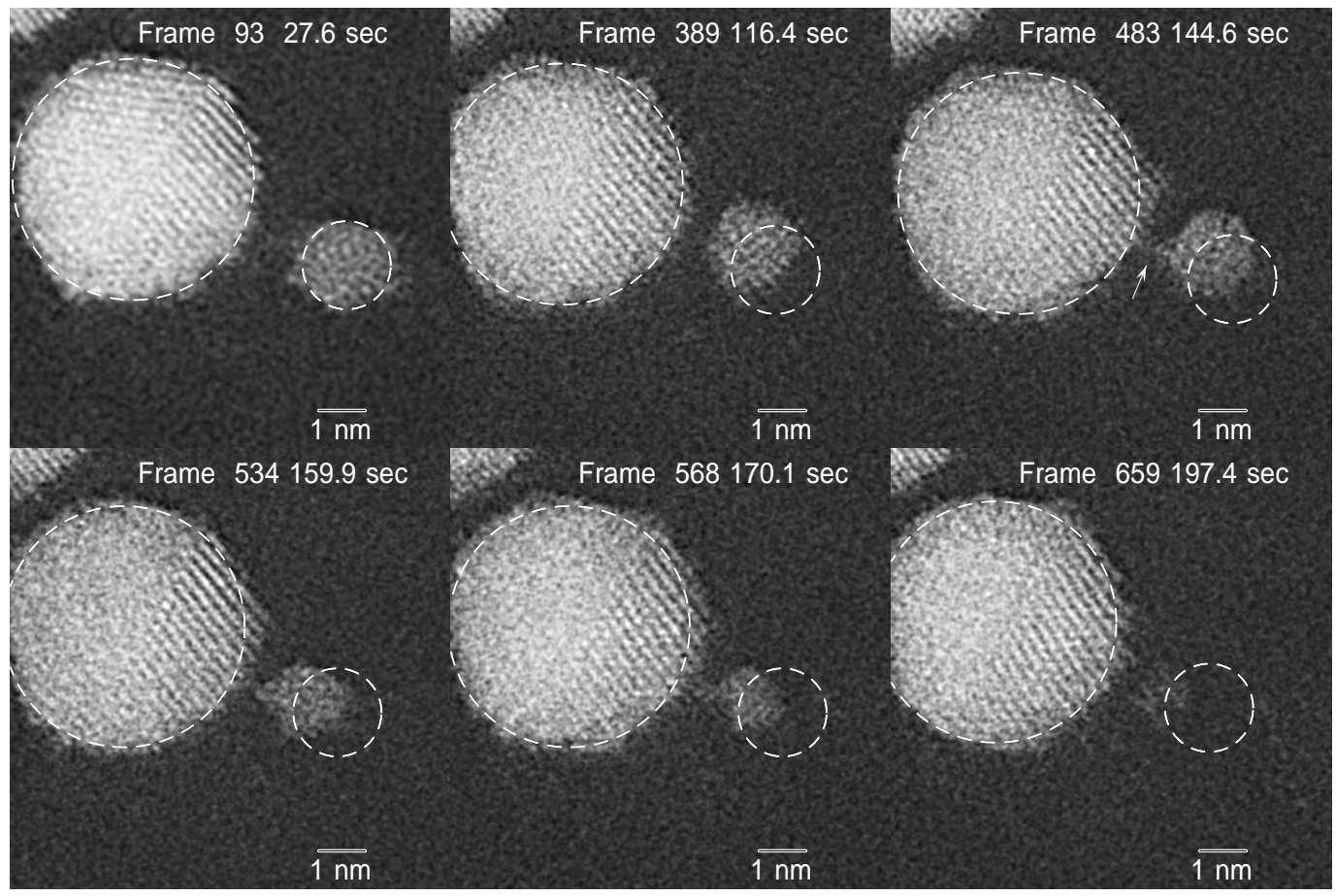

Fig. 2. Observation of coalescence in the presence of a barrier. Material transport occurs through an apparent nanopore, or narrow region with a lowered barrier to diffusion. Initially, the smaller particle is pulled towards the large sphere, but is stopped at a distance of about 0.7-0.8 $\mathrm{nm}$. Atomic transport then continues in a narrow region (arrow), until most of the small particle has been transported to the larger particle. 